\title{
Insights into Substrate Binding and Catalysis in Bacterial Type I Dehydroquinase
}

María MANEIRO,${ }^{\ddagger}$ Antonio PEÓN,${ }^{\ddagger}$ Emilio LENCE,${ }^{\ddagger}$ José M. OTERO,${ }^{\dagger}$ Mark J. VAN RAAIJ,${ }^{\&}$ Paul THOMPSON,${ }^{\varphi}$ Alastair R. HAWKINS ${ }^{\varphi}$ and Concepción GONZÁLEZ-BELLO $*{ }^{\ddagger}$

Centro Singular de Investigación en Química Biológica y Materiales Moleculares (CIQUS), Universidad de Santiago de Compostela, calle Jenaro de la Fuente s/n, 15782 Santiago de Compostela, Spain.

${ }^{\dagger}$ Departamento de Bioquímica y Biología Molecular, Centro Singular de Investigación en Química Biológica y Materiales Moleculares (CIQUS), Universidad de Santiago de Compostela, 15782 Santiago de Compostela, Spain.

${ }^{\&}$ Departamento de Estructura de Macromoléculas, Centro Nacional de Biotecnología (CSIC), Campus Cantoblanco, 28049 Madrid, Spain.

${ }^{\varphi}$ Institute of Cell and Molecular Biosciences, Medical School, University of Newcastle upon Tyne, Newcastle upon Tyne NE2 4HH, UK.

Corresponding Author Information:

*Prof. Dr. Concepción González-Bello, Centro Singular de Investigación en Química Biológica y Materiales Moleculares (CIQUS), Universidad de Santiago de Compostela, calle Jenaro de la Fuente s/n, 15782 Santiago de Compostela, Spain. Tel: +34 881815726; FAX: +34 881815704; e-mail: concepcion.gonzalez.bello@usc.es

Key words: dehydroquinase, X-ray crystallography, MD simulation, binding, enzymatic mechanism, Salmonella typhi

\begin{abstract}
Structural, biochemical and computational studies to study the substrate binding and the role of the conserved residues of the type I dehydroquinase (DHQ1) enzyme active site are reported. The crystal structure of DHQ1 from Salmonella typhi in complex with (2R)-2-methyl-3-dehydroquinic acid, a substrate analog, was solved at $1.5 \AA$. The studies reported here revealed a previously unknown key role of the conserved Glu46, Phe145 and Met205 residues as well as for Gln236, Pro234, Ala233, with the latter being located in the flexible substrate-covering loop. Gln236 showed to be responsible for the folding of this loop and for the dramatic reduction of its flexibility, which triggers the active site closure. Glu46 proved to be key in bringing the substrate close to the Lys/His catalytic pocket to initiate the catalysis. The present study could be useful in the rational design of inhibitors of this challenging and recognized target for the development of novel herbicides and antimicrobial agents.

Abbreviations used: DHQ1, Type I Dehydroquinase; St-DHQ1, Type I Dehydroquinase from Salmonella typhi, Sa-DHQ1; Type I Dehydroquinase from Staphylococcus aureus; DHQ2, Type II Dehydroquinase; $\mathrm{Hp}$ DHQ2, Type II Dehydroquinase from Helicobacter pylori; Mt-DHQ2, Type II Dehydroquinase from Mycobacterium tuberculosis; Sc-DHQ2, Type II Dehydroquinase from Streptomyces coelicolor; MD, molecular dynamics; LHMDS, lithium bis(trimethylsilyl)amide; TBS, tert-butyldimethylsilyl.
\end{abstract}

\section{INTRODUCTION}

The disruption of the bacterial growth cycle by preventing the synthesis and assembly of key components involved in bacterial processes has proven to be a succesful strategy for the development of novel 
antibacterial drugs [1-3]. Therefore, a detailed knowledge of the catalytic mechanism, the binding determinants and the essential motions of selected enzymes can be valuable for the rational design of new inhibitors that can be used as drugs. In recent years, computational methods have consolidated their value as important complementary tools to the biochemical and structural data that can assist in the detailed knowledge of how the enzymatic reactions are catalyzed. Here we report results from structural, biochemical and computational studies that help to improve our understanding of the binding determinants, the role of essential residues and the structural changes responsible for the formation of the Michaelis complex that triggers the reaction catalyzed by a key enzyme in bacteria.

In recent years, the enzymes involved in the shikimic acid pathway, in which chorismic acid is synthesized, have attracted a great deal of attention as excellent targets for the development of novel herbicides and antimicrobial agents. These enzymes are essential in many pathogenic bacteria (database for essential genes in bacteria see http:www.essentialgene.org) and they are present in fungi, higher plants and certain apicomplexan parasites [4-5], which are the cause of malaria and toxoplasmosis, but are absent in mammals. Particular attention has been paid to the inhibition of the third enzyme of the pathway, namely the dehydroquinase (3-dehydroquinate dehydratase, DHQ, EC 4.2.1.10) that catalyzes the reversible dehydration of 3-dehydroquinic acid (1) to form 3-dehydroshikimic acid (2) (Scheme 1). There are two distinct types of DHQ enzymes, named type I (DHQ1, aroD gene) and type II (DHQ2, aroD/aroQ gene) [67]. In the work reported here we focused on the DHQ1 enzyme, which is present in important pathogenic bacteria such as Salmonella typhi, Staphylococcus aureus, Escherichia coli and Salmonella enterica subsp. enterica serovar typhimurium. The deletion of the aroD gene that encodes the DHQ1 enzyme from $S$. typhi and Shigella flexneri has afforded satisfactory live oral vaccines, with the latter providing monkeys with protection against oral challenge with live S. flexneri 2457T [8-10]. Besides the attractive characteristics of this enzyme, very few inhibitors of the DHQ1 enzyme have been reported to date [11-13].

The reaction catalyzed by the DHQ1 enzyme consists of an overall syn elimination of water involving the loss of the pro- $R$ hydrogen at $\mathrm{C} 2$, with the reaction initiated by the formation of a Schiff base between the C3 carbonyl group of the substrate and an essential Lysine (Lys170 in S. typhi) [14-17]. Once the substrateSchiff base is formed, a base residue of the active site then removes the C2 equatorial hydrogen to afford an enamine, which undergoes acid-catalyzed elimination of the $\mathrm{C} 1$ hydroxyl group - a reaction that is mediated by an essential histidine (His143 in S. typhi) acting as a proton donor. Site-directed mutagenesis and structural studies have shown that His143 is involved in both formation and subsequent hydrolysis of the Schiff base intermediates and is also the base that removes the pro- $R$ hydrogen [17-20]. Computational studies also suggested that the product-Schiff base hydrolysis is the rate-determining step [21-22].

Considering that the active center of DHQ1 is highly conserved (see the Supplementary Figure S1), it is likely that some of these conserved residues might have a role beyond substrate recognition, as suggested previously for Gln236 [23-24], which is located in the flexible covering-substrate $(h H)$ loop that closes over after substrate binding. It would be important to understand the required essential movements of this loop and of the active site conserved residues in embedding the natural substrate into the catalytic pocket of DHQ1 for the formation of the substrate-Schiff base that triggers the enzymatic process. Hence, the availability of crystal structures of the enzyme/substrate analog complexes would provide valuable information that could improve our understanding of this complex enzyme. Reasoning that the reaction catalyzed by DHQ1 involves the abstraction of the equatorial pro- $R$ hydrogen at $\mathrm{C} 2$, the substitution of this hydrogen atom by a methyl group would provide an inhibitor that would be structurally very close to the natural substrate. To this end, $(2 R)$-2-methyl-3-dehydroquinic acid (3) was synthesized (Scheme 1) and the crystal structure of DHQ1 from Salmonella typhi (St-DHQ1) in complex with 3 was solved at $1.50 \AA$. Molecular Dynamics (MD) simulation studies conducted with this structure and the Michaelis complex allowed us to identify a previously unknown key role beyond substrate recognition of several conserved residues. In addition, a detailed knowledge of the substrate binding process is reported. 
<smiles>O=C(O)C1=CC(=O)[C@H](O)[C@H](O)C1</smiles>

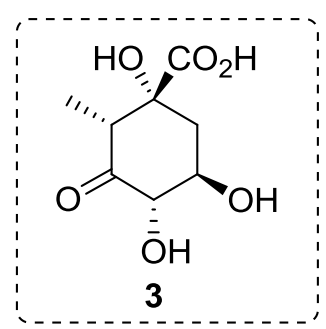

Scheme 1 Enzymatic conversion of 3-dehydroquinic acid (1) to 3-dehydroshikimic acid (2) catalyzed by DHQ1 and target compound 3

\section{EXPERIMENTAL}

\section{Dehydroquinase Assays}

Wild-type DHQ2 from Helicobacter pylori (Hp-DHQ2), Streptomyces coelicolor (Sc-DHQ2), and Mycobacterium tuberculosis (Mt-DHQ2), and DHQ1 from Salmonella typhi (St-DHQ1) and Staphylococcus aureus ( $\mathrm{Sa}$-DHQ1) were purified as described previously [25-28]. Concentrated solutions of Hp-DHQ2 (6.4 $\left.\mathrm{mg} \mathrm{mL}{ }^{-1}\right)$ and $M t$-DHQ2 $\left(1.8 \mathrm{mg} \mathrm{mL}^{-1}\right)$ were stored at $4{ }^{\circ} \mathrm{C}$ in PPB $(50 \mathrm{mM}$, pH 7.2), DTT (1 mM) and $\mathrm{NaCl}(150 \mathrm{mM})$ and PPB $(150 \mathrm{mM}, \mathrm{pH} 6.6)$ and DTT $(1 \mathrm{mM})$, respectively. Sc-DHQ2 $\left(5 \mathrm{mg} \mathrm{mL}^{-1}\right)$ was stored at $4{ }^{\circ} \mathrm{C}$ in Tris/HCl $(20 \mathrm{mM}, \mathrm{pH} 7.5)$ and DTT $(0.5 \mathrm{mM})$. Concentrated solutions of St-DHQ1 (0.85 $\left.\mathrm{mg} \mathrm{mL}^{-1}\right)$ and $S a$-DHQ1 (4.0 $\left.\mathrm{mg} \mathrm{mL}^{-1}\right)$ were stored in PPB $(50 \mathrm{mM}$, pH 6.6 and 7.2 , respectively) and DTT $(1 \mathrm{mM})$ at $-80^{\circ} \mathrm{C}$. When required for assays, aliquots of the enzyme stocks were diluted in water and buffer and stored on ice. Both enzymes were assayed in the forward direction by monitoring the increase in absorbance at $234 \mathrm{~nm}$ in the UV spectrum due to the absorbance of the enone-carboxylate chromophore of 3-dehydroshikimic acid (2) $\left(\varepsilon / \mathrm{M}^{-1} \mathrm{~cm}^{-1} 12000\right)$. For DHQ2, standard assay conditions were $\mathrm{pH} 7.0$ at $25^{\circ} \mathrm{C}$ in Tris/ $\mathrm{HCl}$ or Tris HOAc $(50 \mathrm{mM})$. For DHQ1, pH 7.2 at $25^{\circ} \mathrm{C}$ in PPB $(50 \mathrm{mM})$ was used. Each assay was initiated by addition of the substrate. Solutions of 3-dehydroquinic acid (1) were calibrated by equilibration with DHQ1 or DHQ2 and measurement of the change in the UV absorbance at $234 \mathrm{~nm}$ due to the formation of the enone-carboxylate chromophore of 3-dehydroshikimic acid (2). The initial rates at fixed enzyme and substrate concentrations $\left(0.2-5 K_{\mathrm{m}}\right)$ were measured. Kinetic constants were evaluated using the GraFit 5 program (Erithacus Software Ltd.).

The $K_{\mathrm{i}}$ values of compound $\mathbf{3}$ (see Supplementary Experimental data) against $S t$-DHQ1 and $S a$-DHQ1 were obtained from Dixon plots $(1 / \mathrm{v} v s[\mathrm{I}])$ of assay data. The initial rates at fixed enzyme and substrate concentrations $\left(0.5-1.4 K_{\mathrm{m}}\right)$ were measured in the absence and in the presence of various inhibitor concentrations. The kinetic constants compound 3 against DHQ2 were evaluated as for the natural substrate by monitoring the increase in absorbance at $251 \mathrm{~nm}$ in the UV spectrum due to the absorbance of the enonecarboxylate chromophore of the resulting 2-methyl-3-dehydroshikimic acid.

\section{Sodium borohydride inactivations}

A solution of sodium borohydride $(2 \mu \mathrm{L}, 0.5 \mathrm{M})$ in aqueous sodium hydroxide $(40 \mathrm{mM})$ was added to a 1 $\mathrm{mL}$ incubation of compound $3(0.5 \mathrm{mM})$ and the enzyme (1 unit) in PPB $(50 \mathrm{mM}, \mathrm{pH} 7.2)$ at $25^{\circ} \mathrm{C}$. The DHQ1 activity was assayed before and after addition of sodium borohydride. After the sequential additions of aliquots of sodium borohydride solution, reduction of the enzyme activity was observed but without full 
inactivation. On the contrary, full inactivation was observed for those control experiments carried out in the presence of the natural substrate. In the absence of compound $\mathbf{3}$, no loss of activity was obtained.

\section{Crystallization of $S t$-DHQ1/3 Binary Complex}

A freshly purified solution of St-DHQ1 $\left(0.85 \mathrm{mg} \mathrm{mL}^{-1}\right)$ was concentrated to $8 \mathrm{mg} \mathrm{mL}^{-1}$ in $10 \mathrm{mM}$ Tris/HCl $\mathrm{pH} 7.4,40 \mathrm{mM} \mathrm{KCl}$ and $250 \mathrm{mM}$ solution of compound 3 in methanol was then added at 1:20 (v/v) to give a solution of approximately 10 equivalents of inhibitor per protein monomer. Needle-shaped crystals of up to $0.01 \mathrm{~mm} \times 0.01 \mathrm{~mm}$ of the $S t$-DHQ1/3 binary complex were obtained after 8 weeks of vapor diffusion in sitting drops comprised of $2.0 \mu \mathrm{L}$ of protein/inhibitor solution mixed with $2.0 \mu \mathrm{L}$ of reservoir solution and equilibrated against $0.15 \mathrm{~mL}$ reservoirs containing 32\% (w/v) PEG 4000, 0.1 M citrate-phosphate pH 5.2.

\section{Structure Determination of St-DHQ1/3 Binary Complex}

Crystals were mounted into cryoloops and directly flash frozen by rapid immersion in liquid nitrogen. X-ray diffraction data were collected on beamline BL13-XALOC (Alba Synchrotron, Spain) from crystals maintained at $100 \mathrm{~K}$. The diffraction data were processed, scaled, corrected for absorption effects and the crystal unit-cell parameters were calculated by global refinement using XDS [29], SCALA [30], and other programs from the CCP4 software suite [31]. The structures were solved by molecular replacement using the program MOLREP [32] with a search model generated from PDB entry 1QFE [7]. The structures and geometrical restraints of the ligands were generated with the PRODRG2 server [33] and were manually placed during the model building, which was performed with COOT [34]. Reflections for calculating Rfree [35] were selected randomly, refinement of the models was performed with REFMAC [36] and final structure validation was performed with MOLPROBITY [37]. The data collection, refinement and model statistics are summarized in Supplementary Table S1. Figures depicting structures were prepared using PYMOL (http://www.pymol.org).

\section{RESULTS}

\section{Synthesis of (2R)-2-Methyl-3-dehydroquinic acid (3)}

Compound 3 was obtained by alkylation of the previously described ketone 6 [38] (Scheme 2). Treatment of ketone 6 with lithium bis(trimethylsilyl)amide at $0{ }^{\circ} \mathrm{C}$ followed by reaction with methyl iodide, subsequent deprotection of the TBS-protected groups and hydrolysis of the carbolactone by heating at $50{ }^{\circ} \mathrm{C}$ with $50 \%$ aqueous trifluoroacetic acid gave the desired $(2 R)$-2-methyl derivative 3 . NOE experiments confirmed the $R$ stereochemistry of the $\mathrm{C} 2$ chiral center. Irradiation of $\mathrm{H} 4$ in $\mathbf{3}$ led to enhancement of the signal for $\mathrm{H} 2$ $(1.1 \%)$.

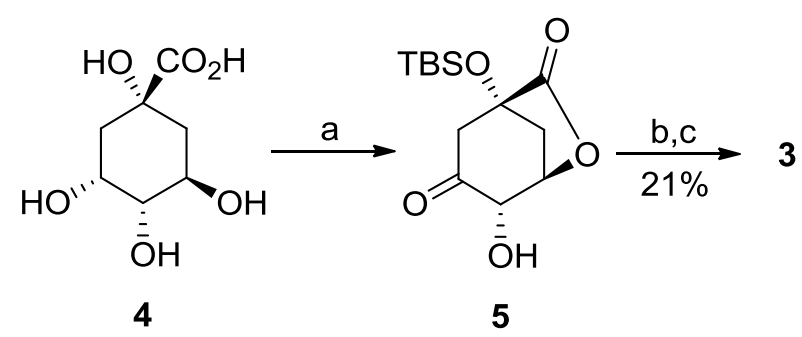

\section{Scheme 2 Synthesis of (2R)-2-methyl-3-dehydroquinic acid}

Reagents and conditions: (a) [38]. (b) 1. LHMDS, 15-crown-5, THF, $0{ }^{\circ} \mathrm{C} .2$. MeI, $0{ }^{\circ} \mathrm{C}$. (c) TFA/ $\mathrm{H}_{2} \mathrm{O}$ $(50 \%), 80^{\circ} \mathrm{C}$.

\section{Enzymatic Assays}


Compound 3 was assayed against $S t$-DHQ1 and $S a$-DHQ1 and was found not to be a substrate for the two enzymes. In addition, treatment of an incubation of both DHQ1 with $\mathbf{3}$ and sodium borohydride did not result in an inactivation of the enzyme, as determined in a subsequent assay with the natural substrate. Therefore, compound $\mathbf{3}$ does not form an imine linkage with this enzyme. As expected, compound $\mathbf{3}$ proved to be a reversible competitive inhibitor of $S t$-DHQ1 and $S a$-DHQ1 with $K_{\mathrm{i}}$ values of $305 \pm 17 \mu \mathrm{M}$ and $282 \pm$ $24 \mu \mathrm{M}$, respectively [39]. In contrast, compound 3 proved to be a weak substrate for the DHQ2 enzyme. This compound has the required axial hydrogen that is removed by an essential tyrosine to afford an enolate intermediate, which undergoes the acid-catalyzed elimination of the $\mathrm{C} 1$ hydroxyl group in a reaction mediated by a conserved histidine [40]. Mt-DHQ2, Hp-DHQ2 and Sc-DHQ2 slowly converted (2R)-2methyl-3-dehydroquinic acid (3) into 2-methyl-3-dehydroshikimic acid. When the kinetic parameters of all three enzymes for compound $\mathbf{3}$ were determined and compared to those measured for the natural substrate, the $K_{\mathrm{m}}$ values for compound $\mathbf{3}$ were found to be lower than that for the natural substrate but marked decreases in the $k_{\text {cat }}$ values to less than $1 \%$ that of the natural substrate were found (Table 1). This change represents a reduction in specificity of $4.6-8.310^{3}$ relative to the natural substrate.

Table 1 Kinetic parameters for the conversion of 3-dehydroquinic acid (1) and (2R)-2-methyl-3-dehydroquinic acid (3) with several DHQ2 enzymes

Results are means \pm S.D. for three observations

\begin{tabular}{lllll}
\hline Enzyme & Compound & $k_{c a t}\left(\mathrm{~s}^{-1}\right)$ & $K_{m}(\mu \mathrm{M})$ & $k_{c a t} / K_{m}\left(\mathrm{~s}^{-1} \mathrm{M}^{-1}\right)$ \\
\hline$M t$-DHQ2 & $\mathbf{1}$ & $154 \pm 7$ & $15 \pm 2$ & 10.27 \\
& $\mathbf{3}$ & $66 \pm 610^{-2}$ & $11 \pm 3$ & 0.06 \\
Hp-DHQ2 $^{\#}$ & $\mathbf{1}$ & $56 \pm 1$ & $448 \pm 21$ & 0.13 \\
& $\mathbf{3}$ & $33 \pm 110^{-2}$ & $247 \pm 22$ & $0.1310^{-2}$ \\
Sc-DHQ2 $^{\#}$ & $\mathbf{1}$ & $3527 \pm 116$ & $263 \pm 24$ & 13.41 \\
& $\mathbf{3}$ & $9 \pm 1$ & $138 \pm 25$ & 0.06 \\
\hline
\end{tabular}

${ }^{\dagger}$ Assay conditions were Tris/acetic acid $(50 \mathrm{mM}, \mathrm{pH} 7.0)$ at $25^{\circ} \mathrm{C}$.

${ }^{\#}$ Assay conditions were Tris/ $\mathrm{HCl}(50 \mathrm{mM}, \mathrm{pH} 7.0)$ at $25^{\circ} \mathrm{C}$.

\section{Structural Studies}

The crystal structure of compound $\mathbf{3}$ in complex with $S t$-DHQ1 was obtained by co-crystallization and was solved at $1.50 \AA$. Crystals were flash-frozen directly from the crystallization mixtures by rapid immersion in liquid nitrogen. Under the crystallization conditions there is sufficient cryo-protectant (PEG) to freeze directly the protein crystals in liquid nitrogen without further manipulation. X-ray diffraction data were collected from crystals cryo-cooled in a stream of cold nitrogen gas $(100 \mathrm{~K})$ at ambient pressure using synchrotron radiation and were then processed. The structure was determined by molecular replacement, using the previously described structure of St-DHQ1 covalently attached to the active site by Lys170, which was obtained by sodium borohydride reduction of the corresponding product-Schiff base intermediate (PDB entry 1QFE) [7] as a search model and the structures were refined. A summary of the statistical data following data reduction and processing is given in Supplementary Table S1.

The St-DHQ1/3 binary complex crystallized with four copies in the asymmetric unit (designated as chains A, B, C and D) as two dimers with helices F, G and H [7] at the dimer interface (Figure 1). The four crystallographically independent copies superimpose well onto each other, with rms differences in the range 0.318-0.218 $\AA$ after superposition of $C^{\alpha}$-atom pairs (Figure 1C). The structure refines with good geometric parameters and clear electron density is visible for all amino acids with the exception of residues 229-234 located on the flexible covering-substrate loop, which are not visible in all chains. Comparison of this crystal structure with PDB entry 1QFE [7] shows that the cyclohexane ring in 3 is displaced by 2.7-3.8 $\AA$ out from the binding site (Figure 1D). Specifically, the oxygen atom of the C3 ketone group in 3 is $3.2-4.2 \AA$ away from the $\varepsilon$-amino group of Lys170, which is located on $\beta$-strand $\mathrm{f}$. For chains $\mathrm{A}, \mathrm{C}$ and $\mathrm{D}$, the binding mode of the ligand is quite similar while for chain $B$ the ligand is located in the farthest position from the Lys 170/His 143 catalytic pocket (4.2 A). Significant differences are also observed in the position of the His143, Phe145 and Gln236 side chains. The latter residue is located in the flexible loop and it is usually in 
close contact with one of the oxygen atoms of the C1 carboxylate group of the substrate and the Schiff base intermediates. A large degree of flexibility in the side chain of His143 is observed while the $\varepsilon$-amino group of the Lys170 residue remains fixed in an extended disposition for all the chains by interaction with the guanidinium group of Arg48 via a bridging water molecule. In view of the ligand binding differences observed in chains A-D for the reported crystal structure, it seems that the ketone derivative $\mathbf{3}$ is entering the active site (chain B). It was considered that this crystal structure provides an excellent opportunity to study in detail the essential enzyme motions for the formation of the Michaelis complex that triggers the enzymatic reaction. To this end, MD simulation studies were carried out and the results are discussed below.

Unbiased, calculated electron density maps showed clear electron density for the ketone derivative 3 (Figures 1D-E and see Supplementary Figure S2). As in PDB entry 1QFE [7], the ligand binds to the active site by a salt-bridge between its carboxylate group and the guanidinium group of Arg213, which is located on the distorted helix $G$ at the subunit interface. The conserved Arg213, which has previously been identified by chemical modification, is the key residue for carboxylate recognition [7]. As the ligand is displaced by 2.7-3.8 $\AA$ away from the binding pocket, the usual bidentate hydrogen bond between the carboxylate group of Glu46 and the C4 and C5 hydroxyl groups is not observed and only the C4 hydroxyl group interacts with Glu46 (2.6 $\AA$ ). As a consequence of this displacement, the guanidinium group of Arg48 and the Ser21 side chain also establish electrostatic and hydrogen bonding interactions with both the $\mathrm{C} 4$ and C5 hydroxyl groups (3.1 $\AA$ each) rather than just with the C5 hydroxyl group. The C3 ketone group in 3 interacts with the guanidinium group of Arg82, the carboxylate group of Glu46 and the $\varepsilon$-amino group of Lys 170 via two bridging water molecules (WAT2019 and WAT2020). Notably, the methyl group of the ligand is located 3.5-5.0 $\AA$ away from the methylsulfanyl group of Met203 and the oxygen atom of the C3 ketone group in 1 is located 4.5-5.4 $\AA$ away from the closest atom of the His143 side chain.

\section{Molecular Dynamics Simulation Studies}

Dynamic behavior of St-DHQ1/3 binary complex - MD simulation studies were performed in order to evaluate whether the side chain of Met203 is the residue that is mainly responsible for avoiding the entrance of the compound in the Lys/His binding pocket and to gain further insights into the dynamic behavior of the aforementioned binding interactions. Four models were created using the enzyme geometries found for chains A-D. Unsolved residues were modeled using the web-based ModLoop server [41]. These MD simulation studies were carried out on the monomer in aqueous solution using the molecular mechanics force field AMBER [42]. On the basis of previous results from preliminary MD simulations and mechanistic considerations, a dual protonation of His143 and neutral Lys170 was considered. In general, the ligand does not undergo significant changes in the active site in the four models. The root-mean-square deviation (rmsd) with respect to the starting structure was calculated for all complexes to examine the temporal changes in the enzyme structure during the $10 \mathrm{~ns}$ of MD simulation (see Supplementary Figure S3). The rmsd values obtained are in the range 0.6-1.3 $\AA$ and these stabilize after about $1.5 \mathrm{~ns}$. As expected, the motions observed were mainly in the substrate-covering loop. The most relevant changes were obtained with the model created from chain B. The calculated binding free energies for compound $\mathbf{3}$ in the four models also gave the highest binding affinity for this latter complex (see Supplementary Table S2). These calculations were performed using the Molecular Mechanics Poisson-Boltzmann Surface Area (MM/PBSA) [43] approach in explicit water (GB) as implemented in Amber. Remarkably, as for the reported $S t$-DHQ1/3 binary complex, the side chain of Met203 remained in close contact with the guanidinium group of Arg213 and the methyl group of the ligand. Displacement of the Met203 side chain to the apolar pocket involving Ile201 and Ala172 residues was not observed, in contrast to the observed in PDB entry 1QFE [7]. This apolar pocket seems to be important during the catalysis because the pro- $R$ hydrogen at $\mathrm{C} 2$ of the natural substrate, which is specifically removed by the enzyme in the syn elimination of water, is located here.

St-DHQ1 Michaelis Menten Complex - The essential enzyme motions for the formation of the Michaelis complex were studied in order to gain an insight into how Gln236, the residues located in the substratecovering loop and other conserved residues of the active site, in addition to Arg213, are involved in correctly positioning the substrate in the Lys170/His143 catalytic pocket to initiate the enzymatic catalysis. 
To this end, the required DHQ1/1 binary complex was generated using the enzyme geometries found in chain $\mathrm{C}$ of the $S t$-DHQ1/3 binary complex and after manual replacement of compound $\mathbf{3}$ by the natural substrate. The models created using the enzyme geometries found in chains A, B and D were also studied but the aforementioned model gave better results for the whole process. These MD simulation studies were carried out in a similar way to those described for the $S t$-DHQ1/3 model discussed previously. Overall, the formation of the DHQ1 Michaelis complex can be organized into three key steps as follows.

Step 1 - Substrate-Covering loop folding: When the DHQ1/1 binary complex was subjected to $30 \mathrm{~ns}$ of dynamic simulation, the results showed that the Gln236 residue is responsible for initiating the folding of the substrate-covering loop through the formation of two strong hydrogen bonds (Figure 2). Firstly, a hydrogen bonding interaction between the carbonyl side chain of Gln236 and the NH main-chain of Ser232 is established (Figures 2A and 2B). A second hydrogen bond is then formed between the NH side chain of Gln236 and the oxygen atom of the Ser232 side chain. This process can be clearly visualized by analyzing the variation of the distances involving these two hydrogen bonds during the whole simulation. The side chain of Gln232 is always in close contact with the carboxylate group of the natural substrate (about $3 \AA$, Figure 3C) while the interaction between the Ser232 side chain and the C5 hydroxyl group does not start after loop folding (Figure 3D). Therefore, the Gln236 side chain is responsible for the initial approach of Ser232 over the active site (Figure 3A) and it then causes the loop folding (Figure 3B). More importantly, as a result of this folding, a key conformational change of the conserved Pro234 and Ala233 residues was observed (Figure 2C). Specifically, the carbonyl group of Pro234 rotates towards the NH main chain of Ala206 and the side chain of Ala233 is launched onto the active site. Both changes allow the formation of extra internal hydrogen bonds in the loop. One hydrogen bond is formed between the carbonyl group of Ala233 and the NH main chain of Gln236 and an intramolecular hydrogen bond is established between the carbonyl and NH main chain of Ala233, which is also in contact with the carbonyl side chain of Gln236 (Figure 2D). These attractive interactions dramatically reduce the flexibility of the loop and this triggers the pushing movements of the loop over the substrate towards the Lys 170/His143 catalytic pocket. Otherwise a to-and-fro movement of the loop is observed and significant displacement of the substrate does not take place.

Step 2 - Pushing the substrate: When this folded and rigid loop conformation is achieved ( 10 ns), the side chain of Ser232 points towards the active site and is in contact with the C5 hydroxyl group of the natural substrate. A large displacement of the substrate inside the active site takes place. Our MD simulation studies showed that the conserved Glu46 is the residue that progressively pushes the substrate, which is held by a salt bridge with Arg213, into the bottom of the active site. By pulling on the C4 hydroxyl group in 1, and with the assistance of the conserved Arg48 residue (Figure 2F), the substrate is finally located in the Lys/His catalytic pocket. As shown in Figure 4A, significant variations in the distances involving the C4 and C5 hydroxyl groups in $\mathbf{1}$ and the two oxygen atoms of the carboxylate group of Glu46 are observed before the C3 carbonyl group is positioned in close contact to Lys170 side chain (Figure 4D). As the substrate progressively moves deeper into the bottom of the active site, the Met203 side chain moves away from the guanidinium group of Arg213 to the apolar pocket that involves the conserved Ala172 and Ile201 residues. This situation imparts additional hydrophobicity to this important part of the active site, in which the removal of the pro- $R$ hydrogen takes place. Analysis of the amino acid sequence in various DHQ1 (see Supplementary Figure S1) reveals that the type of function is conserved at this position (Met, Ile, etc.).

Once the C3 carbonyl group of 1 is well located in the Lys170/His143 catalytic pocket (after $\sim 13 \mathrm{~ns}$ ), the strong hydrogen bond between the carbonyl group of the conserved Pro234 and the NH main chain of Ala206 neatly seals the active site over the substrate (Figure 2B). After $13 \mathrm{~ns}$ of MD simulation, the substrate remains anchored in the active site by a network of hydrogen bonds and electrostatic interactions with the conserved residues of the active site. More importantly, the C3 carbonyl group is now located in close contact with the essential His 143 side chain (Figure 4C) and, to a lesser extent, with the guanidinium group of Arg82. In this arrangement, His143 is ready to act as a Lewis acid by activating and correctly positioning the $\mathrm{C} 3$ carbonyl group of $\mathbf{1}$ for nucleophilic attack by the $\varepsilon$-amino group of the essential Lys 170 , which is located at the opposite face (Figures 4D and 2B). 
A

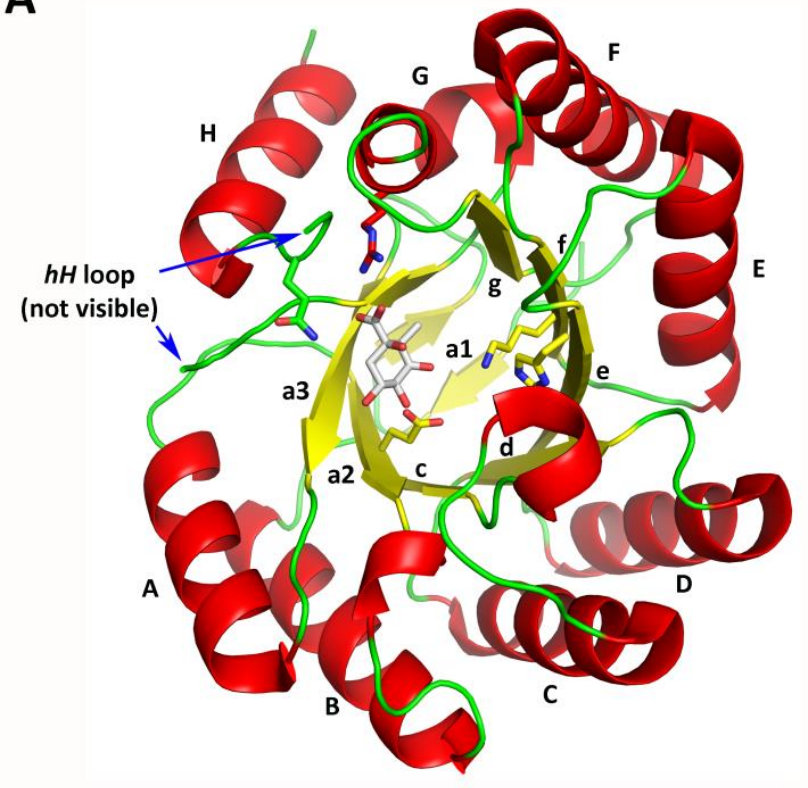

C

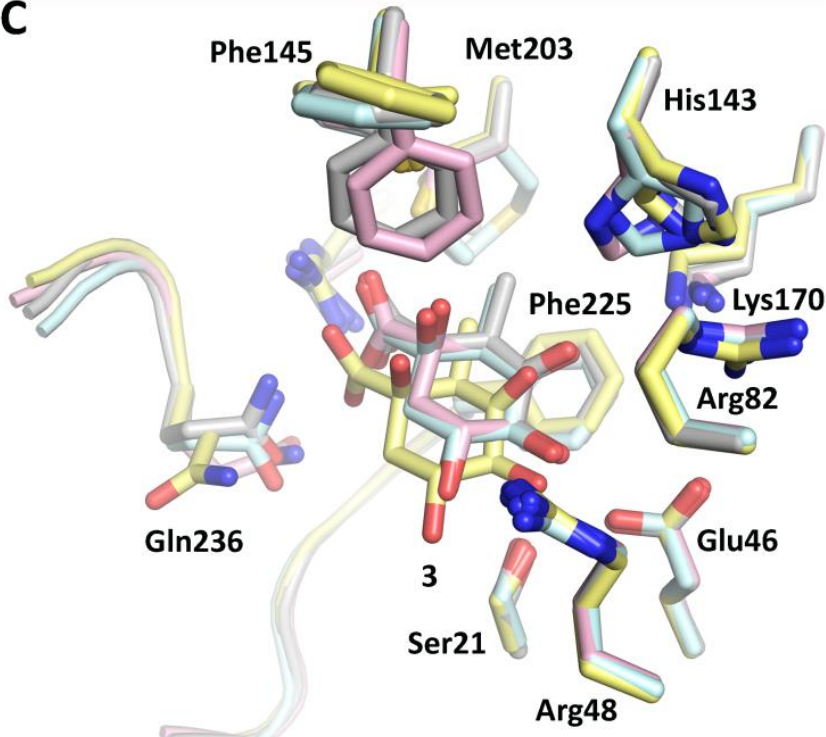

$\mathbf{E}$

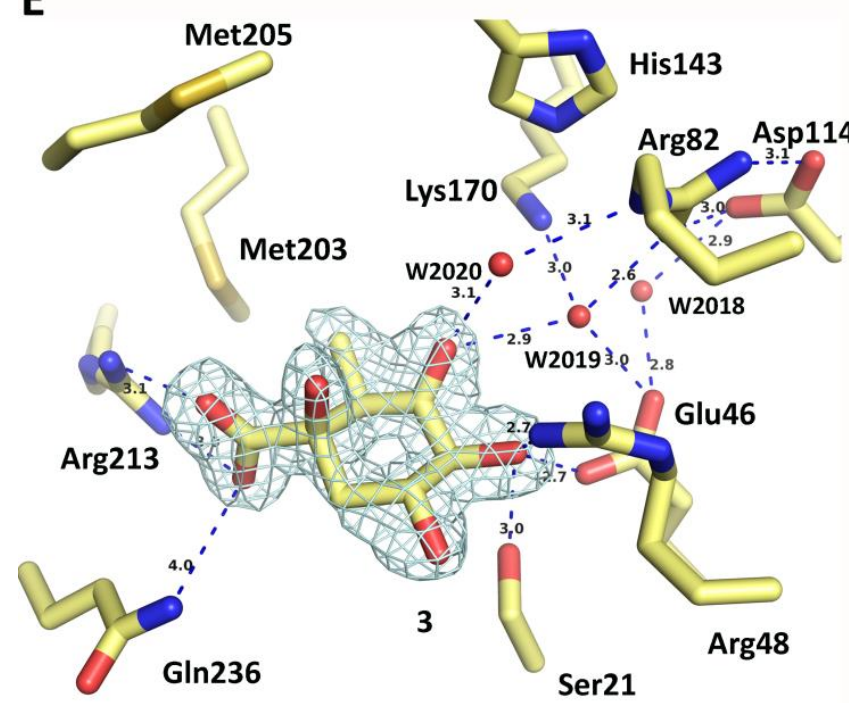

B

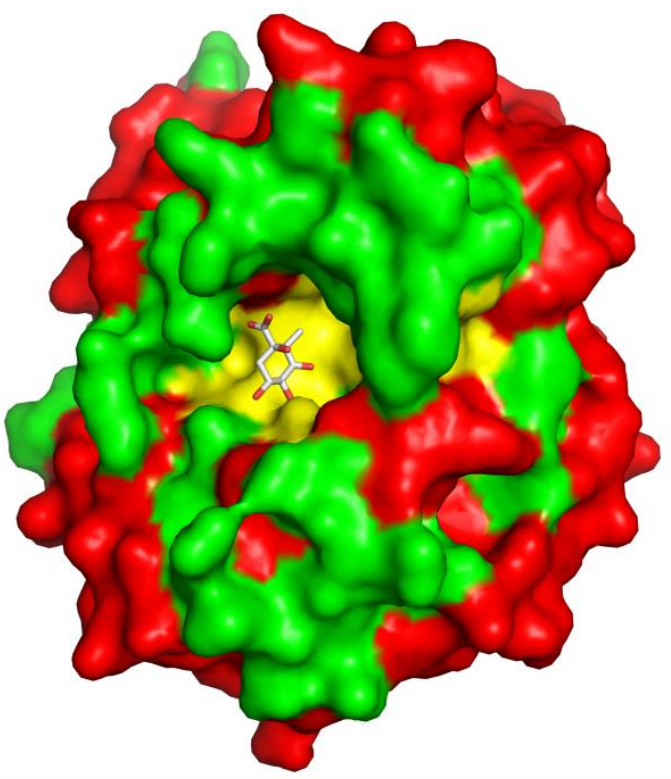

D

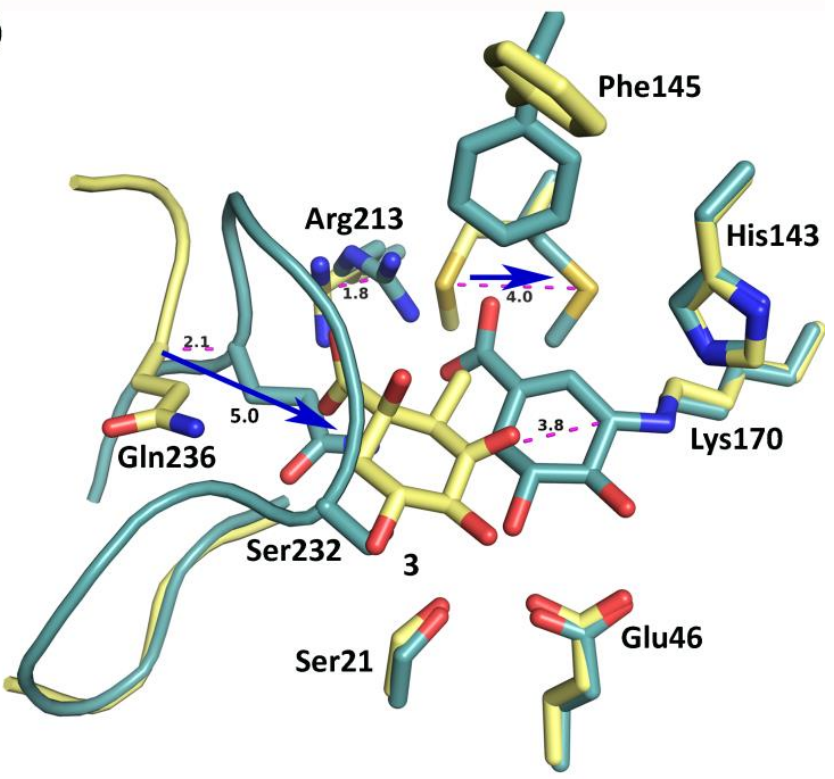

$\mathbf{F}$

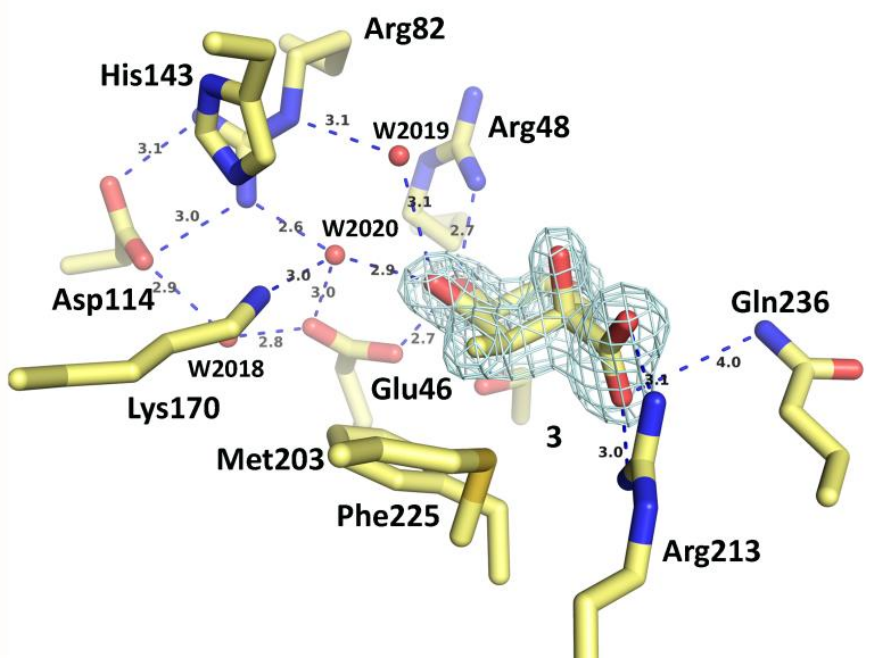

Figure 1 Crystal structure of the $S t$-DHQ1/3 binary complex 
(A) A cartoon of the $S t$-DHQ1/3 crystal structure (chain C), looking into the parallel $\alpha / \beta$ barrel. (B) A surface of the St-DHQ1/3 crystal structure (chain C). (C) Comparison of the binding mode of methyl derivative 3 with St-DHQ1 in chains A (cyan), B (pink), C (yellow) and D (gray). (D) Comparison of the binding mode of methyl derivative 3 (chain C, yellow) in St-DHQ1 with PDB entry 1QFE (blue). Distance differences are shown as dashed purple lines. (E,F) Unbiased electron density and relevant interactions for ligand 3 (cyan) in $S t$-DHQ1/3 (chain C, yellow) binary complex. From the model obtained by molecular replacement and before inclusion of the inhibitor molecule, refinement was performed to obtain unbiased density for the inhibitor molecule and other model changes. A maximum-likelihood weighted $2 F_{\mathrm{o}}-F_{\mathrm{c}}$ map contoured at $1 \sigma$ is shown up to $1.6 \AA$ around the inhibitor molecule (red). The final model including the ligand molecule is superimposed onto the map. Polar interactions between methyl derivative $\mathbf{3}$ and the $S t$ DHQ1 are shown as dashed blue lines. Relevant residues are shown and labeled.

Step 3 - Active site sealing - the lid: Finally, the active site is completely shielded from the solvent environment after 19 ns with the entry of the Phe145 side chain to create a lid. Our MD simulation studies allowed us to identify the key small motion and the residues involved in this sealing process of the active site from the solvent environment. The seal is achieved by a small folding of the loop containing residues 144-151, which joins the $\beta$-strand e and helix F and throws the Phe145 side chain into the active site (Figure $2 \mathrm{G})$. This is triggered by the formation of a key hydrogen bond with a neighboring loop that contains residues 174-178, which joins the $\beta$-strand $f$ and helix G. Specifically, the hydrogen bonding between the main-chain carbonyl group of His 146 and the NH side chain of Gln176 causes a small folding in the Phe145-containing loop and this throws the Phe145 side chain into the active site. In all cases and at this position of the loop containing residues 174-178, the type of function is conserved (Asn, Gln, His) (see Supplementary Figure S1). Moreover, after the entrance of the Phe145 side chain into the active site, the Met205 side chain remains located atop the face with the $S$ atom at a distance of less than $4 \AA$ from the phenyl ring carbon atoms (Figures 2E and 4B).

Remarkably, throughout the whole simulation the $\mathrm{C} 1$ carboxylate group swings slightly. Thus, one of the distances between the oxygen atoms of the $\mathrm{C} 1$ carboxylate group in $\mathbf{1}$ and the nitrogen atoms of the Arg213 guanidinium group is significantly longer than the other, a situation that allows a twisting movement of the $\mathrm{C} 1$ carboxylate group. The relative position of one of the oxygen atoms of the carboxylate group is fixed by Gln236 (hydrogen bonding) and Arg213 (electrostatic) side chains. The other oxygen atom is always in close contact with the $\mathrm{C} 1$ hydroxyl group by a strong intramolecular hydrogen bond.

\section{DISCUSSION}

The reported crystal structure of the $S t$-DHQ1/3 binary complex and the MD simulation studies conducted with this and the Michaelis complex (St-DHQ1/1) created from this crystal structure give an insight into the essential motions of the flexible substrate-covering loop and the binding determinants of St-DHQ1. This information allowed us to identify a previously unknown key role of some residues beyond substrate binding. In view of the ligand binding differences observed in the four chains A-D of the reported StDHQ1/3 binary complex solved at $1.50 \AA$, it seems that the ketone derivative $\mathbf{3}$ is entering the active site but it is unable to reach the Lys170/His143 catalytic pocket. The oxygen atom of the C3 ketone group and the methyl group in 3 are located 4.5-5.4 $\AA$ and 3.5-5.0 $\AA$ away from the closest atom of the His143 side chain and the methylsulfanyl group of Met203, respectively. This fact suggests that the equatorial C2 methyl group in 3 prevents the ligand from becoming embedded in the active site due to the steric hindrance caused by the Met203 side chain. This latter residue is also located in close contact with the guanidinium group of the conserved Arg213 rather than with the apolar pocket of the active site involving Ile201 and Ala172 residues, as observed in PDB entry 1QFE [7]. Therefore, it seems that compound $\mathbf{3}$ is not a substrate for DHQ1, not only because it lacks the equatorial pro- $R$ hydrogen required to be removed by the enzyme but also because it is not even capable of covalently binding to the essential Lys170. Similar results were obtained with $\mathrm{Sa}$-DHQ1 in the enzymatic assays studies. Therefore, the structural data and the results of the 
MD simulation studies suggest that the proximity of the C3 ketone group in 1 to the His143 and a closed conformation of the substrate-covering loop are required for the covalent binding of the substrate to DHQ1, which is essential for catalysis.

A

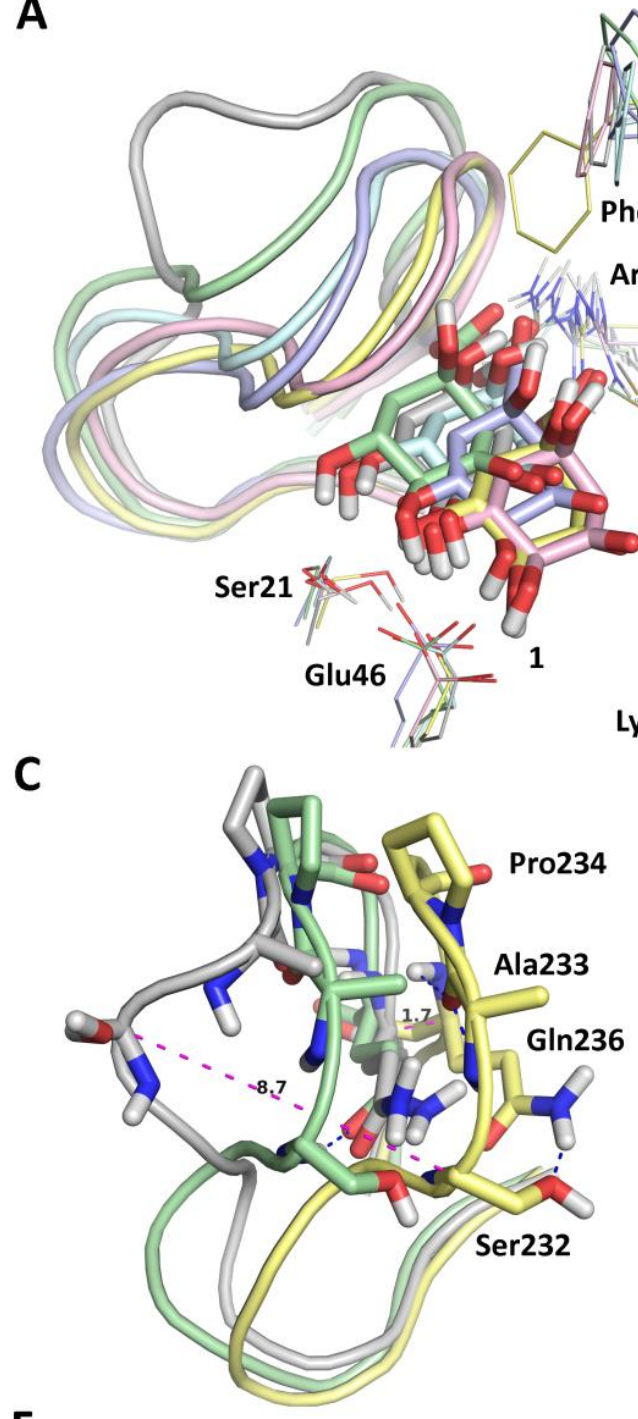

$\mathbf{F}$

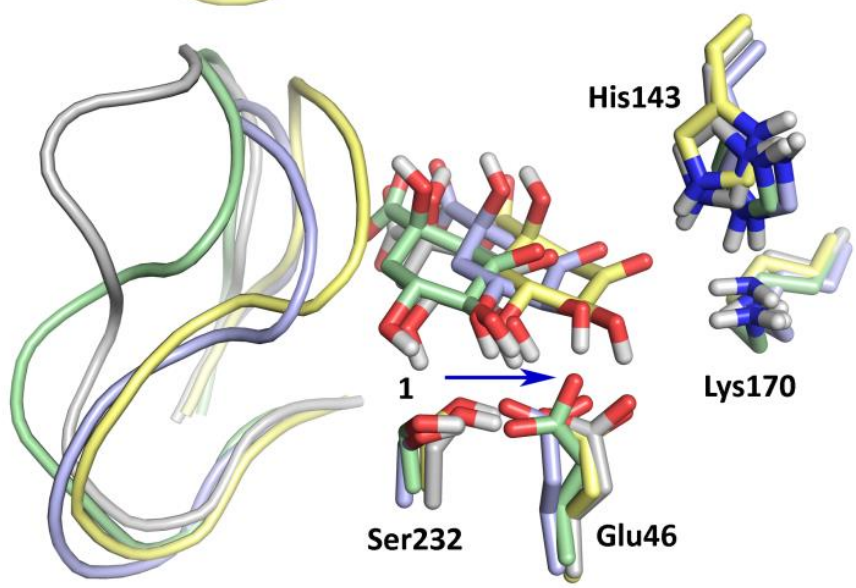

D

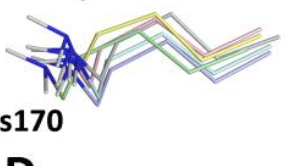

B
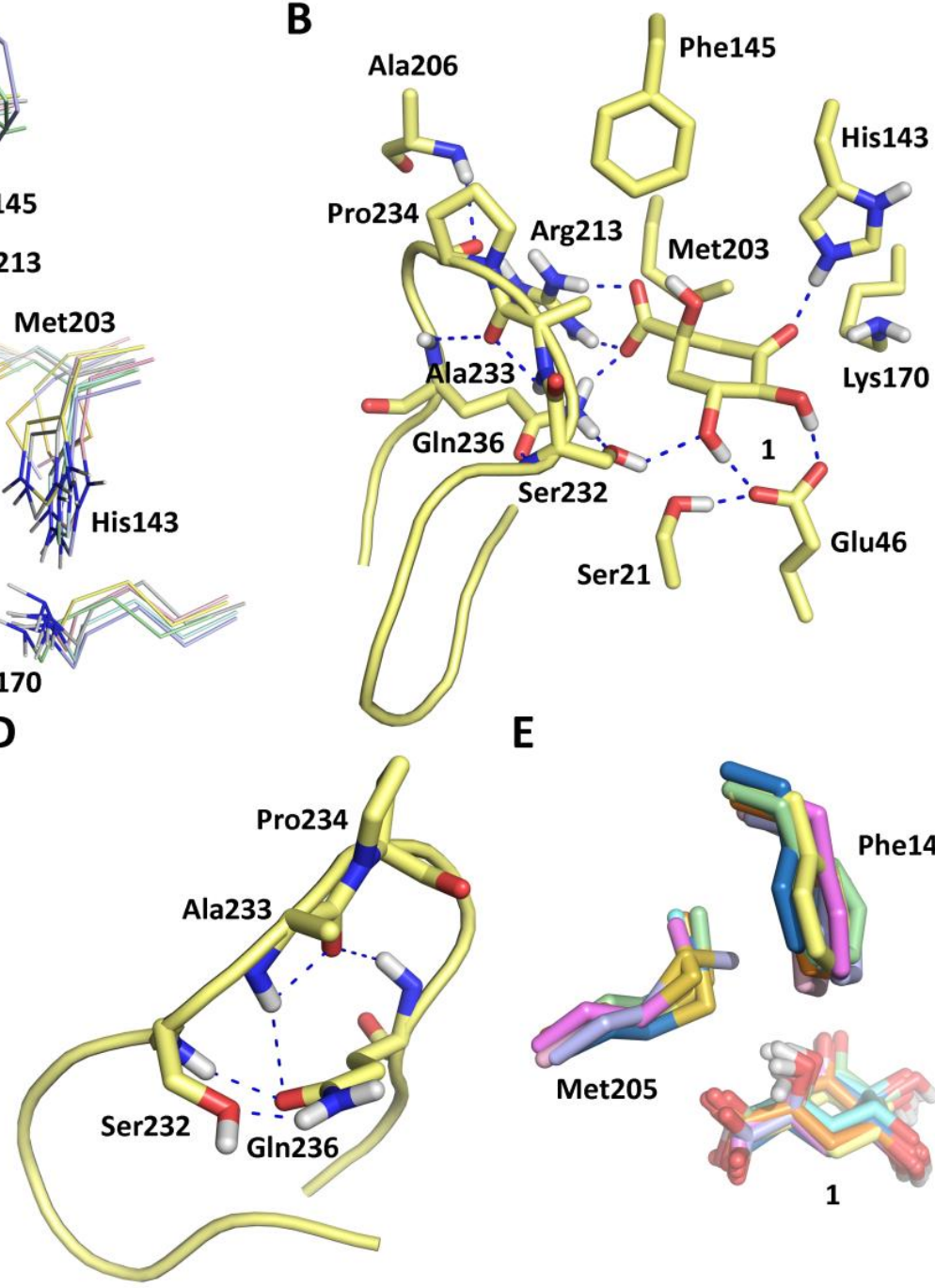

E

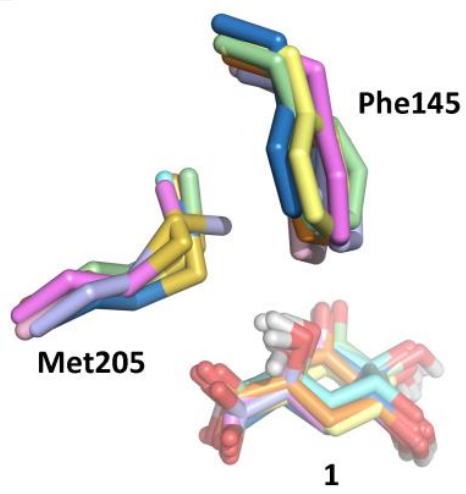

G

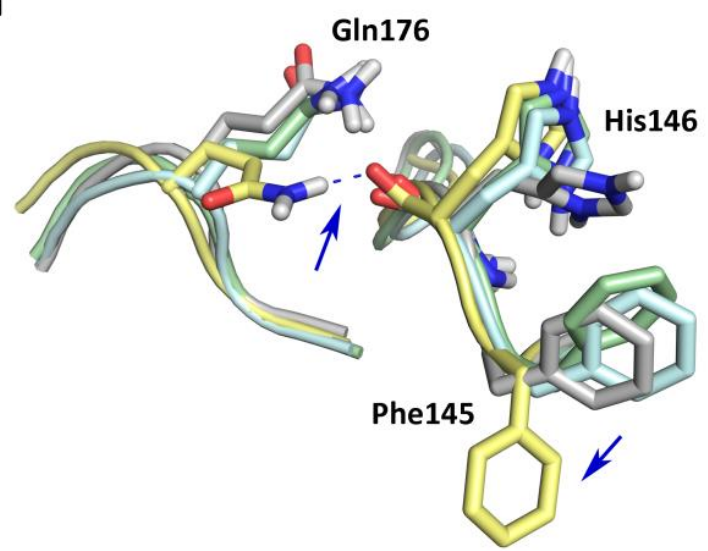

Figure 2 Michaelis complex formation process obtained by MD simulations studies

(A) Comparison of several snapshots of the flexible loop and the position of the natural substrate [0.02 ns (gray), $5 \mathrm{~ns}$ (green), $6 \mathrm{~ns}$ (cyan), $12 \mathrm{~ns}$ (blue), $10 \mathrm{~ns}$ (blue), $18 \mathrm{~ns}$ (pink) and $23 \mathrm{~ns}$ (yellow)]. (B) Michaelis complex that triggers the enzymatic reaction. (C) Closing motion of the covering-substrate loop. (D) Key hydrogen bonding interactions that reduce the loop flexibility. (E) Key contact between the Met205 side 
chain and the aromatic ring of Phe145 that keep Phe145 inside of the active site. (F) Detailed view of how the side chain of conserved Glu46 pulls on the substrate to fit it in the bottom of the active site. $(\mathrm{G})$ Closing motion of the loop that acts as a lid for the active-site-cleft by dropping the Phe145 side chain into the active site. Note how Gln236 is responsible for the loop folding and how the attractive internal hydrogen bonding interactions of the loop result in this fold triggering the pushing process of the substrate. See also how the hydrogen bonding interaction between the side chain carbonyl group of Gln176 and the main-chain carbonyl group of His146 causes the entrance of the Phe145 side chain into the active site. Relevant side chain residues are shown and labeled.

A

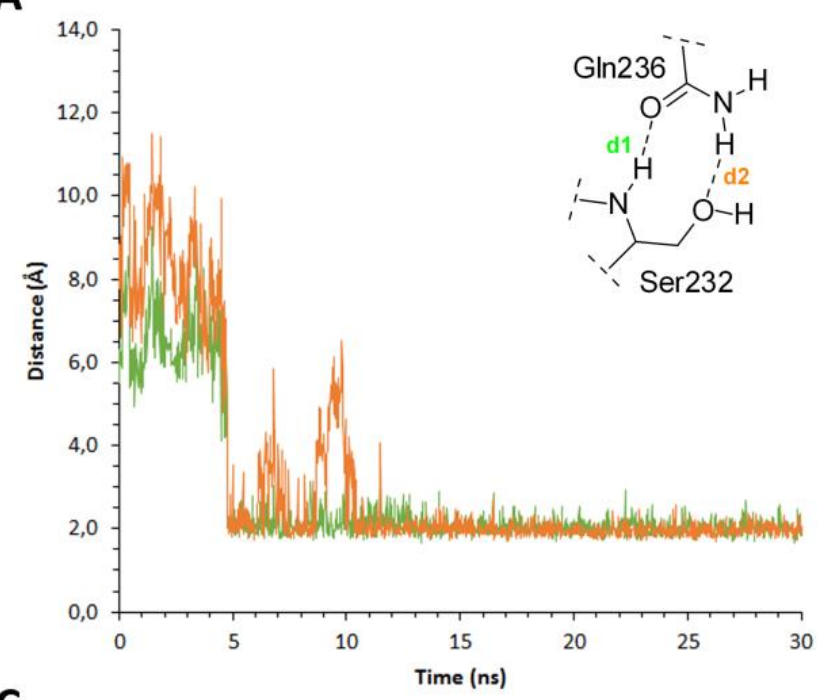

C

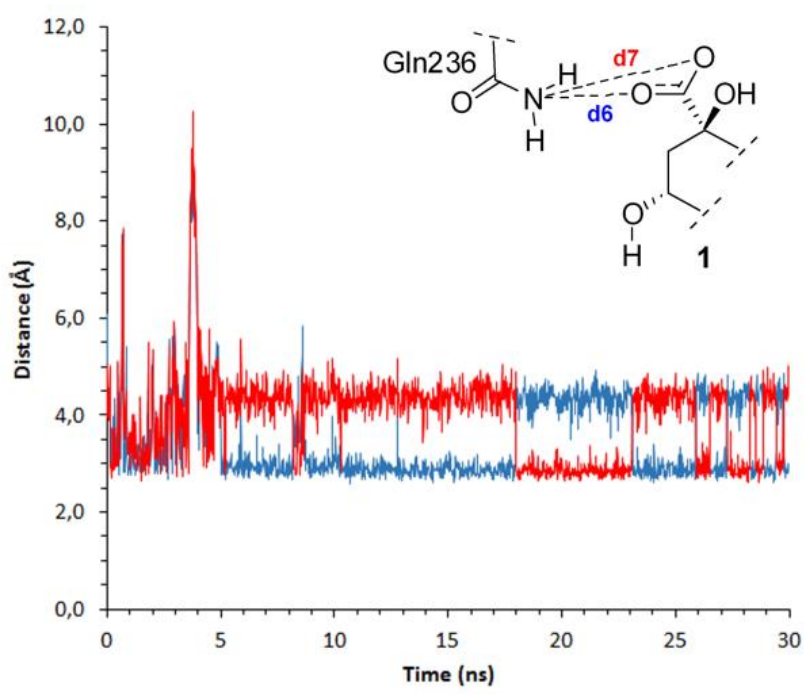

B

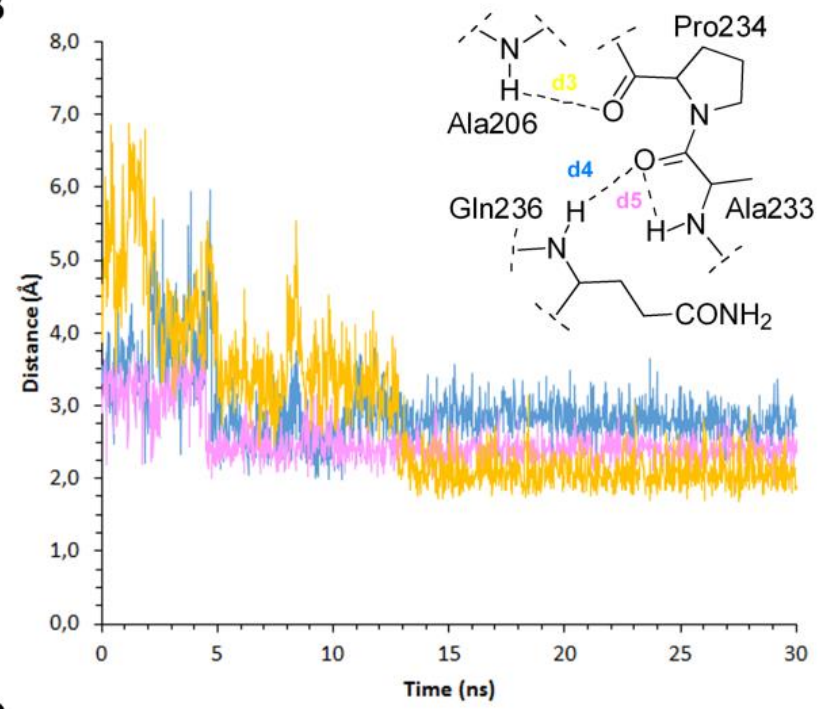

D

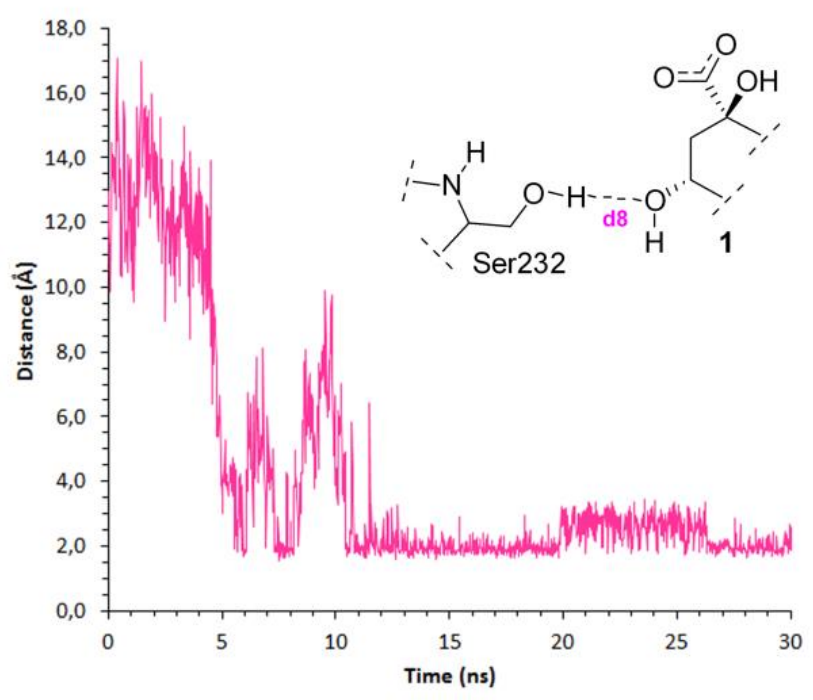

Figure 3 Variation of distances involving residues located in the $h H$ loop obtained by MD simulations studies

(A) Carbonyl side chain (OE1 atom) of Gln236 and $\mathrm{NH}$ (H atom) main chain of Ser232 and hydroxyl side chain (O atom) of Ser232 and NH (1HE2 atom) side chain of Gln236. (B) Carbonyl group of Pro234 and $\mathrm{NH}$ group of Ala206, NH and carbonyl groups of Ala233 and carbonyl group of Ala233 and NH main-chain of Gln236. (C) NE2 atom of Gln236 and the oxygen atoms $(\mathrm{O} 1, \mathrm{O6})$ of $\mathrm{C} 1$ carboxylate group in 1. (D) $\mathrm{C} 5$ hydroxyl group (O atom) in $\mathbf{1}$ and side chain hydroxyl group (H atom) of Ser232. 
A

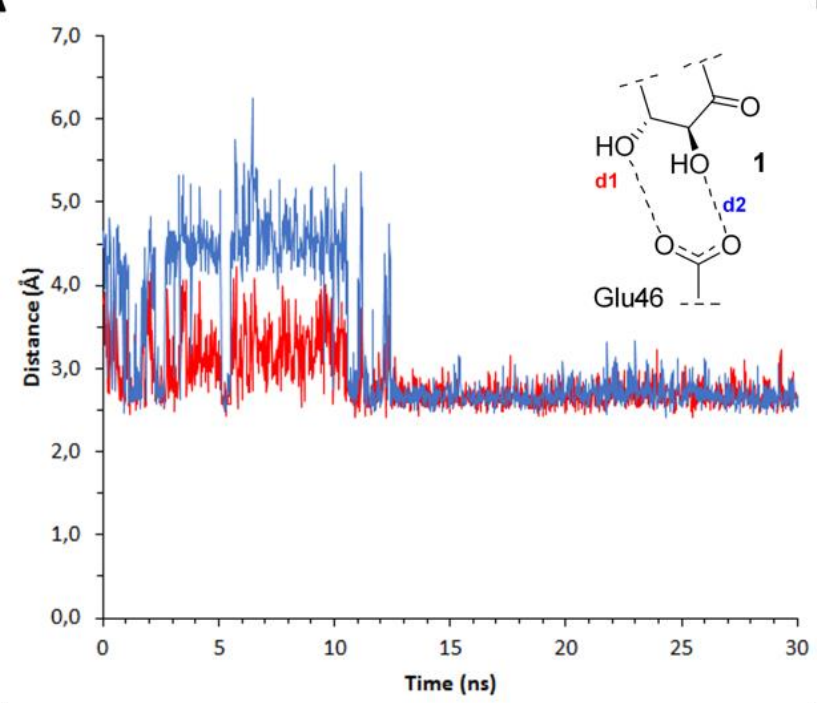

C

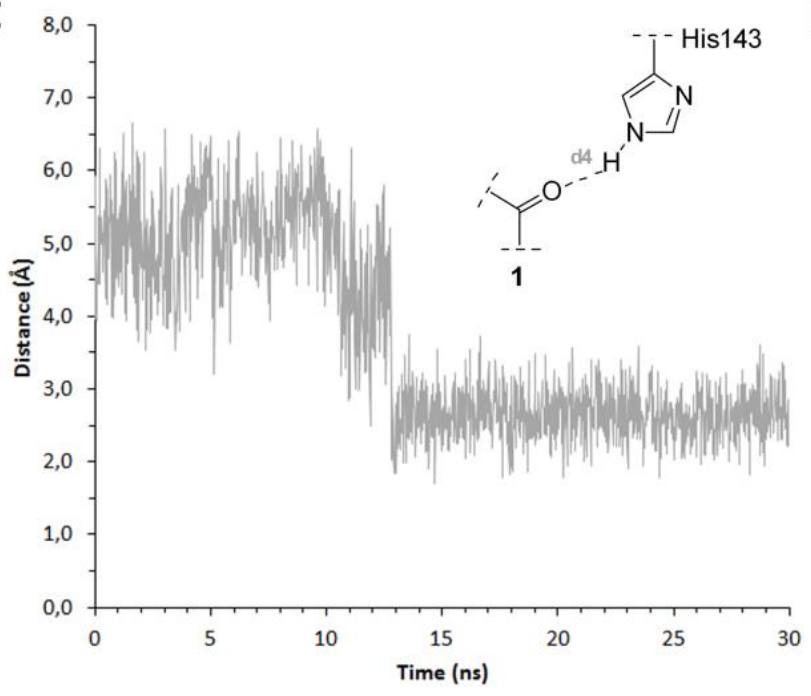

B

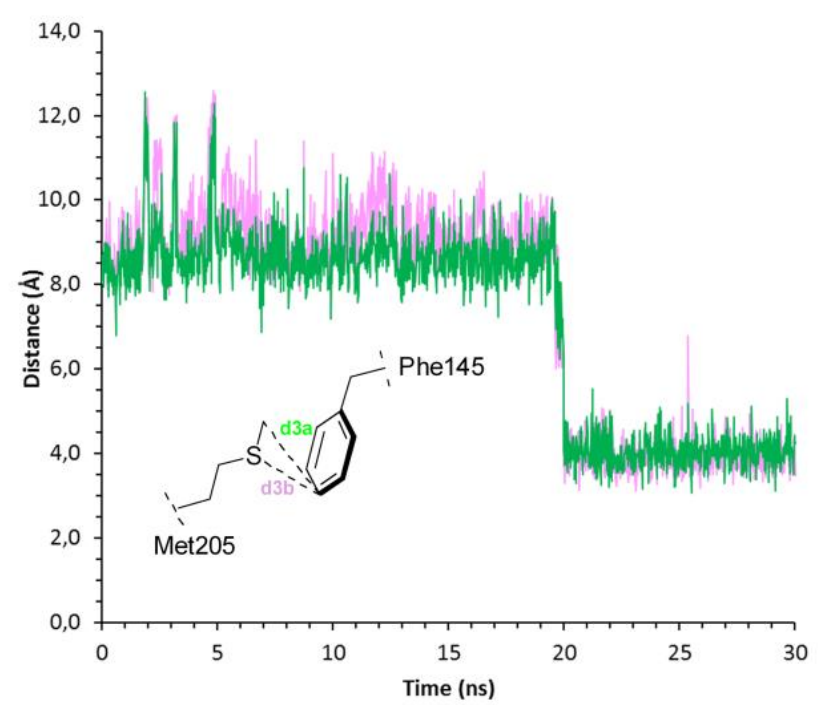

D

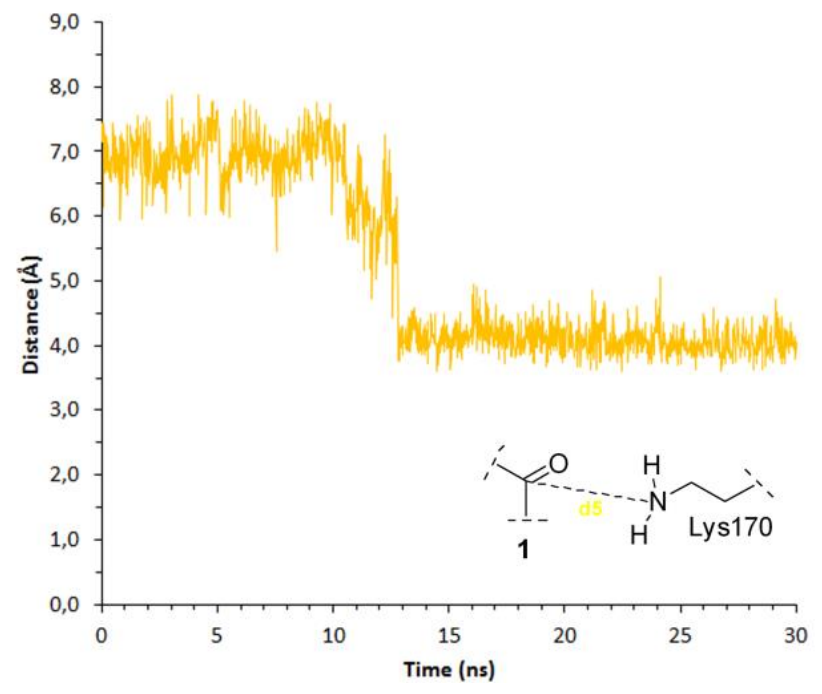

Figure 4 Variation of distances involving conserved Glu46, Met205, Phe145, Lys170 and His143 residues obtained by MD simulations studies

(A) The carboxylate group of Glu46 and C4 and C5 hydroxyl groups in 1. (B) the side chains of Met205 and Phe145. (C) the C3 carbonyl group in $\mathbf{1}$ and His143 side chain. (D) the C3 carbonyl group in $\mathbf{1}$ and Lys170 side chain.

We consider that the reported crystal structure is an excellent structure to study the entrance process of the natural substrate into the active site because (1) it contains a compound that is very similar to the natural substrate, (2) the ligand is located far away from the Lys170/His143 catalytic pocket, (3) the substratecovering loop (not visible) is probably in the open conformation and (4) the variety of loop conformations and models offered by the differences observed in the position of the ligand and of the visible residues of the substrate-covering loop. From this structure, four models were constructed and evaluated. The variety of models studied allowed us to obtain a detailed view of the formation process of the Michaelis complex in a process that involves three key steps: (1) folding of the substrate-covering loop, (2) pushing the substrate and (3) active site sealing.

The results obtained from our MD simulation studies on the St-DHQ1 Michaelis complex suggest that the conserved Gln236 residue has a crucial role in the first step. In particular, it is responsible for initiating the folding of the substrate-covering loop by forming key hydrogen bonding interactions involving residues 
Gln236, Ala233 and Ser232. These attractive interactions dramatically reduce the flexibility of the loop and allow its closure over the substrate.

Based on structural and site-directed mutagenesis studies, Light et al. [24] suggested a more extensive role of Gln236 than merely binding the substrate. These studies were carried out with the S232A and Q236A DHQ1 enzyme variants from Salmonella typhimurium LT2 and the results showed a $\sim 30$-fold increase in $K_{\mathrm{m}}$ values in both cases and marked differences in their corresponding $k_{\text {cat }}$ values. In particular, 50- and 1000fold reductions in specificity relative to the natural substrate were obtained, respectively. Moreover, Harris et al. [44] reported that removal of the C5 hydroxyl group in 1 caused a 1000-fold reduction in specificity relative to the natural substrate. The removal of both $\mathrm{C} 4$ and C5 hydroxyl groups, which interact with the carboxylate group of Glu46 through a bidentate hydrogen bond, provided a compound that is not a substrate for DHQ1 and is enable to form an imine-bound species. We consider that our results also support the more extensive role of Gln236 that has been suggested and also highlighted a previously unknown role of Glu46 beyond the recognition of the $\mathrm{C} 4$ and $\mathrm{C} 5$ hydroxyl groups. By pulling on the C4 hydroxyl group in 1, and with the assistance of the conserved Arg48 residue, the conserved Glu46 progressively pushes the substrate into the bottom of the active site with the assistance of the folded and rigid loop.

The results obtained from the MD simulation studies also provided a detailed view of the sealing of the active site by the entry of the Phe 145 side chain inside the site to create a lid. This process is the last step in the formation of the Michaelis complex and it does not occur until the active site is neatly closed by the substrate-covering loop. In order to achieve this closure the enzyme undergoes a small folding of the loop containing residues 144-151. This is caused by a hydrogen bonding interaction between the main-chain carbonyl group of His146 and the NH side chain of Gln176. These studies also highlight the key role of the Met205 beyond creating an additional hydrophobic layer on top of the loop together with Phe145 and Ala172, which might explain why it is conserved. This residue seems to be involved in keeping the Phe145 side chain inside the active site by an attractive interaction derived from its location at the edge of the phenyl ring, an interaction that is widely found in numerous crystal structures [45-46]. Our MD simulation studies also suggest that one of the oxygen atoms of the $\mathrm{C} 1$ carboxylate group in $\mathbf{1}$ is involved in correctly positioning the $\mathrm{C} 1$ hydroxyl group to accept a proton from the conserved His143. This situation is markedly different for DHQ2. In the latter case, the $\mathrm{C} 1$ hydroxyl group is correctly positioned during the dehydration step by a conserved asparagine residue of the active site [40].

In conclusion, the results of the present study show that the replacement of the equatorial pro- $R$ hydrogen in 3-dehydroquinic acid (1), the natural substrate of the reaction catalyzed by the third enzyme of the shikimic acid pathway, by a methyl group provides a week irreversible competitive inhibitor of DHQ1 and a poor substrate for DHQ2. A reduction in specificity for the latter of 4.6-8.3 $10^{3}$ relative to the natural substrate was found. The inhibition of DHQ1 is not only due to the lack of the equatorial pro- $R$ hydrogen required to be removed by the enzyme, but also to the steric hindrance caused in the apolar pocket of the active site involving residues Met203, Ala172 and Ile201. This type of compound does not form an imine linkage with the enzyme, which is required for turnover, and leads to an open conformation of the substrate-covering loop, as shown in the crystal structure of St-DHQ1/3 binary complex solved at $1.5 \AA$.

Our results on the formation of the St-DHQ1 Michaelis complex provide strong evidence that the process involves the following steps: (1) folding of the substrate-covering loop triggered by Gln236; (2) reduction of the loop flexibility by a series of attractive hydrogen bonding interactions involving Pro234, Ala233, Ser232 and Gln236 residues; (3) pushing the substrate into the bottom of the active site, which involves Arg213 and Glu46 residues and the loop; (4) sealing of the active site with the entrance of the Phe145 side chain into the active site after a small folding of the loop containing residues 144-151, a process that is triggered by a hydrogen bonding interaction with the neighboring loop that contains residues 174-178; and (5) capture of the Phe145 side chain inside of the active site by the Met205 residue. These findings reveal a previously unknown key role of these conserved residues and provide details of the required motions of the binding process. Blocking these essential motions and the residues involved in this process could be a useful strategy in the structure-based design of novel inhibitors of this challenging and attractive bacterial target. 


\section{ACCESSION NUMBERS}

Coordinates and structure factors have been deposited in the Protein Data Bank with accession code 4CNO.

\section{AUTHORS CONTRIBUTION}

María Maneiro synthesized the ligand and crystallized the reported binary complex. Antonio Peón and Emilio Lence performed the MD simulation studies. José M. Otero and Mark J. van Raaij carried out X-ray diffraction data collection and solved the structure. Paul Thompson and Alastair R. Hawkins carried out the production and purification of DHQ enzymes. Concepción González-Bello carried out the enzymatic assays, supervised the whole work and wrote the paper.

\section{ACKNOWLEDGMENTS}

We thank the Centro de Supercomputación de Galicia (CESGA) for generous allowance on the Finis Terrae computer. We also thank the ALBA, synchrotron beamline BL13-XALOC (Barcelona, Spain), for the provision of beam time. We thank Professor A.J. Lapthorn (University of Glasgow, Glasgow, U.K.) for providing the $S c$-DHQ2 enzyme for the enzymatic assays.

\section{FUNDING}

This work was supported by the Spanish Ministry of Science and Innovation (grant number SAF201015076) and via FPU fellowships to M.M. and A.P., the Xunta de Galicia (grant number GRC2013/041) and via postdoctoral fellowships to E.L. and J.M.O., and by the European Regional Development Fund (ERDF).

\section{REFERENCES}

1 Walsh, C. (2003). Antibiotics: actions, origins, resistance. ASM Press, Washington, D. C.

2 Rasko, D. A. and Sperandio, V. (2010). Anti-virulence strategies to combat bacteria-mediated disease. Nature Rev. Drug Discov. 9, 117-128.

3 Clatworthy, A. E., Pierson, E., and Hung, D. T. (2007). Targeting virulence: a new paradigm for antimicrobial therapy. Nature Chem. Biol. 3, 541-548.

4 Roberts, F., Roberts, C. W., Johnson, J. J., Kyle, D. E., Krell, T., Coggins, J. R., Coombs, G. H., Milhous, W. K., Tzipori, S., Ferguson, D. J., Chakrabarti, D., and McLeod, R. (1998). Evidence for the shikimate pathway in apicomplexan parasites. Nature 393, 801-805.

5 Campbell, S. A., Richards, T. A., Mui, E. J., Samuel, B. U., Coggins, J. R., McLeod, R., and Roberts, C. W. (2004). A complete shikimate pathway in Toxoplasma gondii: an ancient eukaryotic innovation. Int. J. Parasit. 34, 5-13.

6 Kleanthous, C. K., Deka, R., Davis, K., Kelly, S. M., Cooper, A., Harding, S. E., Price, N. C., Hawkins, A. R., and Coggins, J. R. (1992). A comparison of the enzymological and biophysical properties of two distinct classes of dehydroquinase enzymes. Biochem J. 282, 687-695.

7 Gourley, D. G., Shrive, A. K., Polikarpov, I., Krell, T., Coggins, J. R., Hawkins, A. R., Isaacs, N. W., and Sawyer, L. (1999). The two types of 3-dehydroquinase have distinct structures but catalyse the same overall reaction. Nat. Struct. Biol. 6, 521-525.

8 Tacket, C. O., Hone, D. M., Curtiss III, R., Kelly, S. M., Losonsky, G., Guers, L., Harris, A. M., Edelman, R., and Levine, M. M. (1992). Comparison of the safety and immunogenicity of $\triangle$ aroC $\triangle a r o D$ and $\Delta c y a \Delta c r p$ Salmonella typhi strains in adult volunteers. Infection and Immunity 60 , 536-541.

9 Karnell, A., Cam, P. D., Verma, N., and Lindberg, A. A. (1993). AroD deletion attenuates Shigella flexneri strain $2457 \mathrm{~T}$ and makes it a safe and efficacious oral vaccine in monkeys. Vaccine 11, 830-836. 
10 Tacket, C. O., Hone, D. M., Losonsky, G. A., Guers, L., Edelman, R., and Levine, M. M. (1992). Clinical acceptability and immunogenicity of CVD 908 Salmonella typhi vaccine strain. Vaccine 10, 443-446.

11 González-Bello, C., Harris, J. M., Manthey, M. K., Coggins, J. R., and Abell, C. (2000). Irreversible inhibition of type I dehydroquinase by substrates for type II dehydroquinase. Bioorg. Med. Chem. Lett. 10, 407-409.

12 Bugg, T. D. H., Abell, C., and Coggins, J. R. (1998). Affinity labelling of E. coli dehydroquinase. Tetrahedron Lett. 29, 6783-6786.

13 Ratia, K., Light, S. H., Antanasijevic, A., Anderson, W. F., Caffrey, M., and Lavie, A. (2014). Discovery of selective inhibitors of the Clostridium difficile dehydroquinate dehydratase. PLoS ONE 9, e89356-e89363.

14 Chauduri, C., Ducan, K., Graham, L. D., and Coggins, J. R. (1991). Identification of the active-site lysine residues of two biosynthetic 3-dehydroquinases. Biochem. J. 275, 1-6.

15 Shneier, A., Kleanthous, C., Deka, R., Coggins, J. R., and Abell, C. (1991). Observation of an imine intermediate on dehydroquinase by electrospray mass spectrometry. J. Am. Chem. Soc. 113, 9416-9418.

16 Harris, J., Kleanthous, C., Coggings, J. R., Hawkins, A. R., Abell, C. (1993). Different mechanistic and stereochemical courses for the reactions catalyzed by type I and type II dehydroquinases. J. Chem. Soc., Chem. Commun. 1080-1081.

17 Leech, A. P., James, R., Coggins, J. R., and Kleanthous, C. (1995). Mutagenesis of active site residues in type I dehydroquinase from Escherichia coli. Stalled catalysis in a histidine to alanine mutant. J. Biol. Chem. 270, 25827-25836.

18 Krell, T., Horsbourgh, M. J., Cooper, A., Kelly, S. M., and Coggins, J. R. (1996). Localization of the active site of type II dehydroquinases. Identification of a common arginine-containing motif in the two classes of dehydroquinases. J. Biol. Chem. 271, 24492-24497.

19 Deka, R. K., Kleanthous, C., and Coggins, J. R. (1992) Identification of the essential histidine residue at the active site of Escherichia coli dehydroquinase. J. Biol. Chem. 267, 22237-22242.

20 Light, S. H., Minasov, G., Shuvalova, L., Duban, M. E., Caffrey, M.; Anderson, W. F., and Lavie, A. (2011). Insights into the mechanism of type I dehydroquinate dehydratases from structures of reaction intermediates. J. Biol. Chem. 286, 3531-3539.

21 Yao, Y. and Li, Z.-S. (2012). New insights into the mechanism of the Schiff base hydrolysis catalyzed by type I dehydroquinate dehydratase from S. enterica: a theoretical study. Org. Biomol. Chem. 10, 7037-7044.

22 Yao, Y. and Li, Z.-S. (2012). The reaction mechanism for dehydratation process catalyzed by type I dehydroquinate dehydratase from Gram-negative Salmonella enterica. Chem. Phys. Lett. 519-520, 100-104.

23 Light, S. H., Antanasijcvic, A., Krishna, S. N., Caffrey, M., Anderson, W. F., and Lavie, A. (2014). Crystal structures of type I dehydroquinate dehydratase in complex with quinate and shikimate suggest a novel mechanism of Schiff base formation. Biochemistry 53, 872-880.

24 Light, S. H., Minasov, G., Shuvalova, L., Peterson, S. N., Caffrey, M., Anderson, W. F., and Lavie, A. (2011). A conserved surface loop in type I dehydroquinate dehydratases positions an active site arginine and functions in substrate binding. Biochemistry 50, 2357-2363.

25 Prazeres, V. F. V., Sánchez-Sixto, C., Castedo, L., Shuh, S. W., Lamb, H., Hawkins, A. R., Cañada, F. J., Jiménez-Barbero, J., and González-Bello, C. (2008). Competitive inhibitors of Helicobacter pylori type II dehydroquinase: synthesis, biological evaluation, and NMR studies. ChemMedChem 3, 756770.

26 Gourley, D. G., Coggins, J. R., Isaacs, N. W., Moore, J. D., Charles, I. G., and Hawkins, A. R. (1994). Crystallization of a type II dehydroquinase from Mycobacterium tuberculosis. J. Mol. Biol. 241, 488491.

27 Moore, J. D., Hawkins, A. R., Charles, I. G., Deka, R., Coggins, J. R., Cooper, A., Kelly, S. M., and Price, N. C. (1993). Characterization of the type I dehydroquinase from Salmonella typhi. Biochem. J. 295, 277-285. 
28 Nichols, C. E., Lockyer, M., Hawkins, A. R., and Stammers, D. K. (2004). Crystal structures of Staphylococcus aureus type I dehydroquinase from enzyme turnover experiments. Proteins 56, 625-628.

29 Kabsch, W. (2010). XDS. Acta Cryst. D66, 125-132.

30 Evans, P. (2006). Scaling and assessment of data quality. Acta Cryst. D62, 72-82.

31 Winn, M. D. (2003). An overview of the CCP4 project in protein crystallography: an example of a collaborative project. J. Synchrotron Radiat. 10, 23-25.

32 Vagin, A., and Teplyakov, A. (1997). MOLREP: an automated program for molecular replacement. J. Appl. Cryst. 30, 1022-1025.

33 Schüttelkopf, A. W., and van Aalten, D. M. F. (2004). PRODRG: a tool for high-throughput crystallography of protein-ligand complexes. Acta Cryst. D60, 1355-1363.

34 Emsley, P., and Cowtan, K. (2004). Coot: model-building tools for molecular graphics. Acta Cryst. D60, 2126-2132.

35 Brünger, A. T. (1997). Free R value: cross-validation in crystallography. Methods Enzymol. 277, 366-396.

36 Murshudov, G. N., Vagin, A. A., and Dodson, E. J. (1997). Refinement of macromolecular structures by the maximum-likelihood method. Acta Cryst. D53, 240-255.

37 Davis, I. W., Leaver-Fay, A., Chen, V. B., Block, J. N., Kapral, G. J., Wang, X., Murray, L. W., Arendall, W. B. $3^{\text {rd }}$, Snoeyink, J., Richardson, J. S., and Richardson, D. C. (2007). MolProbity: all-atom contacts and structure validation for proteins and nucleic acids. Nucleic Acids Res. 35, W375-W383.

38 Sánchez-Sixto, C., Prazeres, V. F. V., Castedo, L., Lamb, H., Hawkins, A. R., and González-Bello, C. (2005). Structure-based design, synthesis and biological evaluation of inhibitors of Mycobacterium tuberculosis type II dehydroquinase. J. Med. Chem. 48, 4871-4881.

39 Under assay conditions (50 mM PPB pH 7.2, $25^{\circ} \mathrm{C}$ ) (a) For St-DHQ1: $K_{m}=24 \pm 3 \mu \mathrm{M}, k_{\text {cat }}=1.1 \pm 0.1$ $\mathrm{s}^{-1}$; (b) For $S a$-DHQ1: $K_{m}=18 \pm 3 \mu \mathrm{M}, k_{\text {cat }}=255 \pm 12 \mathrm{~s}^{-1}$.

40 Coderch, C., Lence, E., Peón, A., Lamb, H., Hawkins, A. R., Gago, F., and González-Bello, C. (2014). Mechanistic insight into the reaction catalyzed by bacterial type II dehydroquinases. Biochem. J. 458, 547-557.

41 Fiser, A., and Sali, A. (2003). ModLoop: automated modeling of loops in protein structures. Bioinformatics 19, 2500-2501.

42 Cornell, W. D., Cieplak, P., Bayly, C. I., Gould, I. R., Merz, K. M., Ferguson, D. M., Spellmeyer, D. C., Fox, T., Caldwell, J. W., and Kollman, P. A. (1995). A second generation force field for the simulation of proteins, nucleic acids, and organic molecules. J. Am. Chem. Soc. 117, 5179-5197.

43 Miller III, B. R., McGee Jr., T. D., Swails, J. M., Homeyer, N., Gohlke, H., and Roitberg, A. E. (2012). MMPBSA.py: an efficient program for end-state free energy calculations. J. Chem. Theory Comput. 8, 3314-3321.

44 Harris, J. M., Watkins, W. J., Hawkins, A. R., Coggins, J. R., and Abell, C. (1996). Comparison of the substrate specificity of type I and type II dehydroquinases with 5-deoxy- and 4,5-dideoxydehydroquinic acid. J. Chem. Soc., Perkin Trans. 1, 2371-2377.

45 Salonen, L. M., Ellermann, M., and Diederich, F. (2011). Aromatic rings in chemical and biological recognition: energetics and structures. Angew. Chem. Int. Ed. 50, 4808-4842.

46 Cardomí, A., Gómez-Tamayo, J. C., Gigoux, V., and Fourmy, D. (2013). Sulfur-containing amino acids in 7TMRs: molecular gears for pharmacology and function. Trends Pharmacol. Sci. 34, 320-331. 